\title{
Investigation of vaginal microbiota in sexually active women using hormonal contraceptives in Pakistan
}

\author{
Yasmeen Faiz Kazi ${ }^{*}$, Sobia Saleem ${ }^{1}$ and Nasreen Kazi ${ }^{2}$
}

\begin{abstract}
Background: Previous studies report association of contraceptives with moderate increase in urinary tract infection among sexually active premenopausal women. The aim of our study was to find out whether the use of hormonal contraceptives has any effect on microbiota of the vagina in the contraceptives users in Khairpur Sindh Pakistan.

Methods: A prospective study in woman population of Khairpur Sindh Pakistan aged 20-30 years and 31-40 years, using Hormonal contraceptives was carried out. High vaginal swab samples $(n=100)$ were collected from the test populations as well as control group $(n=100)$ and investigated for vaginal microbial flora using standard microbiological and biochemical techniques.

Results: Vaginal swabs culturing from hormonal contraceptives users in the age group 20-30 years showed statistically insignificant Candida sp (10\% samples), and statistically significant $(p<0.05)$ Staphylococcus saprophyticus. (18\% samples), Streptococcus agalactiae (23\% samples), Escherichia coli (28\% samples) and Lactobacillus fermentum (32\% samples). In the age group 31-40 years, statistically significant percentage of samples $(p<0.05)$ showed Lactobacillus fermentum (28\%), Candida sp (24\%), and E. coli, (24\%) where statistically insignificant samples showed Staphylococcus saprophyticus (13\%) and Streptococcus agalactiae (11\%).

Conclusions: The use of hormonal contraceptives alters the normal microbiota of vagina in women according to the age. Lactobacillus fermentum appeared as the predominant species followed by E. coli among the age group of 20-30 years and, Lactobacillus fermentum, Candida sp and E. coli as predominant among women of age group 31-40 years when compared to corresponding control groups. An inverse relationship between E. coli and Lactobacillus fermentum was observed in the women aged 20-30 years.
\end{abstract}

\section{Background}

Hormonal contraception (HC) mentions to birth control methods that act on the endocrine system.

Previous studies identified urinary tract infection (UTI) as a complication of contraceptive use [1,2]. Around 17 million women per year in the United States suffer UTI and costs billions to the economy [3]. Methods of contraception have suffered a set back in improving the health of women because of concerns in many countries about the mechanism and side effects of the contraceptives [4]. As the population is growing most rapidly in the developing countries including Pakistan, it has put much emphasis to its 'population

\footnotetext{
*Correspondence: yfkazi@yahoo.com; yasmeen.kazi@salu.edu.pk 'Department of Microbiology, Shah Abdul Latif University, Khairpur, Sindh, Pakistan

Full list of author information is available at the end of the article
}

planning prograrmme and despite spending a significant budget, the objectives are yet not achieved [5]. One of the reasons is fear of UTI, a serious health problem affecting millions of women each year. UTI are the second most common type of infection. Women are especially prone to UTI and one woman in five develops a UTI during her lifetime [1]. UTI as a side effect of $\mathrm{HC}$ usage has not been previously documented in Khairpur. This study is aimed at addressing this gap in knowledge by analyzing effect of hormonal contraceptives on vaginal microbiota of women of reproductive age using $\mathrm{HC}$ in Khairpur city.

\section{Methods}

The samples were randomly collected from non-pregnant pre- menopausal women aged $20-30$ and $31-40$ years ( $\mathrm{n}=100$ for each group) using hormonal contraceptions

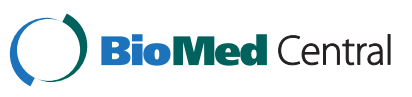


and from control group (women not using contraceptives; $\mathrm{n}=100$ for each group). The samples were taken from different hospitals of Khairpur city, Sindh Pakistan. The hospital include: Civil Hospital, Lady Wellington Hospital, Reproductive Health Services and Maternity Home Luqman. An informed consent was taken prior to the sample collection. This study was approved by the District health committee Khairpur.

\section{High vaginal swab collection}

Vaginal swabs specimens from contraceptive users and control group were collected using commercial sterile cotton swabs by inserting approximately 1 inch into vagina and immediately transported to the laboratory in Amies transport medium (Chem Malaysia) by keeping them in ice box. Microbiological investigations were carried out in the Diagnostic and research center department of Microbiology Shah Abdul Latif University Khairpur. The swabs were aseptically streaked on various culture media such as Blood agar (Oxoid) containing $\mathbf{1 0 \%}(\mathrm{v} / \mathrm{v})$ defibrinated sheep blood, Chocolate agar (Oxoid), Mac Conkey's agar (Oxoid), Nutrient agar (Oxoid) and Saboureaud dextrose agar (Oxoid) and incubated aerobically at $37^{\circ} \mathrm{C}$ for $24 \mathrm{~h}$. After incubation, results were recorded semi-quantitatively as described by Gupta et al. [6] as 0 (no growth), 1+ (colonies on first streak zone), 2+ (colonies on first and second streak zone, $3+$ (colonies on first, second and third streak zone) and 4+ (colonies on first, second, third and fourth streak zone). Colony morphology of isolates was inspected and recorded. Gram stain and biochemical tests were performed using standard microbiological techniques. Pure cultures of the isolated microorganisms were preserved in sterile glycerol broth ( $16 \% \mathrm{v} / \mathrm{v}$ in nutrient broth) for identification by standard biochemical tests. E. coli was initially isolated on Mac Conkey's agar and then in nutrient agar and identified using API-20 E (bioMerieux France) according to manufacturer's instructions. Staphylococcus sp. was grown in nutrient agar and identified on the bases of catalase test, coagulase test, hemolysis on blood agar containing $10 \%(\mathrm{v} / \mathrm{v})$ defibrinated sheep blood and novobiocin (Hugo diagnostics; 5 micro gram disc) sensitivity test using disc diffusion method; Streptococcus sp. was grown in nutrient agar and identified on the bases of catalase test, hemolysis on sheep blood agar containing $10 \%$ (v/v) defibrinated sheep blood, bile solubility test and bacitracin (Oxoid) sensitivity test. Lactobacillus sp. was grown in nutrient agar ( $\mathrm{pH}$ 5.5) in the presence of $5 \% \mathrm{CO}_{2}$ and then identified on the bases of catalase test, and glucose fermenting activity [7-10].

\section{Germ tube test}

Oval budding cells were investigated for identity as Candida albicans by germ tube test [9]. A $500 \mu \mathrm{l}$ of human serum was placed in to a small test tube to observe tube like outgrowths from the cells (known as germ tube), indicative of C. albicans. Using a sterile wire loop, the serum was inoculated with a yeast colony from SDA plate. The tube was placed in a water bath at $37^{\circ} \mathrm{C}$ for $3 \mathrm{~h}$. The wet mount preparation was examined using the $10 \mathrm{X}$ and $40 \mathrm{X}$ objectives with the condenser iris diaphragm closed sufficiently to give good contrast.

\section{Statistical analysis}

The data were analyzed using the Statistical Package for Social Sciences (SPSS) version 10 and the Microsoft Excel (MS) software program. The proportions of microorganisms with age group of $\mathrm{HC}$ user and control group were calculated as a percentage. The degree of association of each type of microorganism from $\mathrm{HC}$ user of each age group with use of hormonal contraception were determined using Mc Nemar's test. Tables were constructed to present the results. Statistical significance was set at the 95\% confidence level CI, confidence interval) or at a $p$-value of less than or equal to 0.05 ( $p$ value $\leq 0.05$ ).

\section{Results}

Vaginal swabs specimen of contraceptive users from different hospitals in Khairpur city were investigated in both age groups and control group. Gram staining showed G + oval budding cells, $G+$ cocci in bunches, $G+$ cocci in chains, G- coccobacilli, and G + filamentous rods. The isolated microorganisms were identified by biochemical tests (Table 1). Further identification of E. coli was performed by API-20E. Staphylococcus sp. was further characterized on the bases of coagulase test, hemolysis on blood agar and novobiocin sensitivity. Confirming of the specie was carried out using novobiocin sensitivity test. The Staphylococcus sp. was coagulae- negative; showed beta hemolysis on blood agar and was resistant to novobiocin (zone size $<17 \mathrm{~mm}$ ) therefore it was identified as Staphylococcus saprophyticus. The Streptococcus sp. showed catalase negative reaction, beta hemolysis on blood agar and was bile insoluble. Bacitracin resistance response indicated that this organism was Streptococcus agalactiae which belong to group B. The Lactobacillus sp. showed catalase negative reaction and fermented glucose with acid and gas which characterized this organism as Lactobaccilus fermentum. In case of Candida sp. further characterization was not performed as the interest was to isolate $C$. albicans which was ruled out by negative germ tube test. Semiquantitative grading and isolation percentage was calculated for test and control groups. Tables 2 and 3 show the semi quantitative grading and isolation percentages from women aged 20-30 and 31-40 years using $\mathrm{HC}$ and from control group respectively. The data showed statistically significant difference in the trend of isolation and semiquantitative grading of vaginal bacteria among the age 
Table 1 Initial biochemical characterization of the vaginal swab isolates from women using hormonal contraceptives and control group

\begin{tabular}{|c|c|c|c|c|c|c|}
\hline \multirow[t]{2}{*}{ Strain } & \multirow[t]{2}{*}{ Isolated microorganism } & \multirow[t]{2}{*}{ Germ tube test } & \multicolumn{3}{|c|}{ Biochemical tests } & \multirow[t]{2}{*}{ Identified microorganism } \\
\hline & & & Catalase & Oxidase & Coagulase & \\
\hline 1 & G+ oval budding cells & Negative & N/A & $\mathrm{N} / \mathrm{A}$ & N/A & Candida sp. \\
\hline 2 & $\mathrm{G}+$ cocci in bunches & $\mathrm{N} / \mathrm{A}$ & + & - & - & Staphylococcus saprophyticus. \\
\hline 3 & $G+$ cocci in chains & $\mathrm{N} / \mathrm{A}$ & - & - & - & Streptococcus agalactiae. \\
\hline 4 & G- coccobacilli & $\mathrm{N} / \mathrm{A}$ & + & - & - & E. coli \\
\hline 5 & G- filamentous rods & $\mathrm{N} / \mathrm{A}$ & - & - & - & Lactobacillus fermentum \\
\hline
\end{tabular}

Vaginal swabs were obtained from women using hormonal contraceptives and processed. The table shows the isolated and identified microorganisms from the age groups: $20-30$ years and 31-40 years ( $n=100$ for each group). N/A denotes 'not applicable'.

group 20-30 years when compared to the control group. Staphylococcus saprophyticus. was found in $18 \%$ of women using $\mathrm{HC}$ and $9 \%$ of the controls $(p<0.05)$, Streptococcus agalactiae in $23 \%$ and $10 \%$ of control $(p<0.05)$, E. coli in $28 \%$ and $6 \%$ of control $(p<0.05)$, Lactobacillus fermentum in $32 \%$ women and $12 \%$ of control $(p<0.05)$. Only the isolation percentages of Candida sp from $\mathrm{HC}$ users and control shown statistically insignificant difference $(p<0.05)$ and the trend remained similar.

In the women of age group 31-40 years, the trend of isolation showed statistically significant difference $(p<0.05)$ among $\mathrm{HC}$ users and respective control group. Here the Candida sp was present in $24 \%$ women and $10 \%$ of control $(p<0.05), E$. coli in $24 \%$ women and $4 \%$ of control $(p<0.05)$ and Lactobacillus fermentum in $28 \%$ women and $16 \%$ of control $(p<0.05)$. Statistically insignificant difference was found between HC users and control for the isolated Staphylococcus and Streptococcus species. Trends of isolation rate (\%) revealed that Lactobacillus fermentum was the predominant vaginal flora in women of age group 20-30 years, where the Candida sp was predominant in women of age group 31-40 years as shown in Figure 1. The control group showed similar trend in both age groups (20-30, 31-40 years) as shown in Figure 2.

\section{Discussion}

This study was undertaken to investigate the effect of oral contraceptives on vaginal ecology of the users of two age groups. Five microbial strains were isolated and evaluated as reference index strains. So far, there is no known prospective study that has ascertained the role of contraceptives in women of different age groups using hormonal contraceptives in Khairpur Sindh Pakistan.

In present study, we report that the overall percentage of vaginal microbiota amongst the contraceptive users of age group 20-30 and 31 to 40 years was high (45\%) and this finding is consistent with other researchers [6,11-13]. The reason why the study was confined to the microbial species reported here is that these microorganisms represent opportunistic group (except Lactobacillus fermentum) and etiology of most urinary tract infections have been attributed to these microbiota.

Our study revealed that there was insignificant difference in type and trend of microorganisms among both control groups. Whereas, a significant difference was observed in the trend of microorganisms particularly for Candida sp and Lactobacillus fermentum between age groups 20-30 and 31-40 years that indicates the role of age factor in vaginal ecology. Though Lactobacillus fermentum was isolated from both age groups, the preventive role remained uncertain in the presence of other species isolated in our study. Lactobacillus fermentum probiotic strains have been used with poor results in urogenital infection [14], and this may have been the cause of overgrowth of the other microorganisms. However, racial

Table 2 Determination of the percentage of microorganisms isolated from vaginal swabs of women using hormonal contraceptives

\begin{tabular}{|c|c|c|c|c|}
\hline \multirow[t]{2}{*}{ Strain } & \multirow{2}{*}{ Cultural characters } & \multirow{2}{*}{$\begin{array}{c}\text { Identified } \\
\text { Microorganisms }\end{array}$} & \multicolumn{2}{|c|}{ Total percentage (\%) in various age groups } \\
\hline & & & $20-30$ years & $31-40$ years \\
\hline 1 & Oval budding cells & Candida sp. & $10(1+)$ & $24(3+)$ \\
\hline 2 & $\mathrm{G}+\mathrm{cocci}$ & Staphylococcussaprophyticus. & $18(2+)$ & $13(1+)$ \\
\hline 3 & $\mathrm{G}+$ cocci in chains & Streptococcus agalactiae & $23(3+)$ & $11(1+)$ \\
\hline 4 & G- coccobacilli & E. coli & $28(4+)$ & $24(3+)$ \\
\hline 5 & $\mathrm{G}+$ long filamentous rods & Lactobacillus fermentum & $32(2+)$ & $28(4+)$ \\
\hline
\end{tabular}

Vaginal swabs were obtained from women using hormonal contraceptives and processed. The table shows the percentages and semi-quantitative grading in parenthesis of isolated and identified microorganisms from age groups: $20-30$ years and 31-40 years and control groups( $\mathrm{n}=100 \mathrm{for}$ each group). 
Table 3 Determination of the percentage of microorganisms from vaginal swabs of control group

\begin{tabular}{|c|c|c|c|c|}
\hline \multirow[t]{2}{*}{ Strain } & \multirow[t]{2}{*}{ Cultural characters } & \multirow[t]{2}{*}{ Identified Microorganisms } & \multicolumn{2}{|c|}{ Total percentage $(\%)$ in various age groups } \\
\hline & & & $20-30$ & $31-40$ \\
\hline 1 & Oval budding cells & Candida sp. & $13(1+)$ & $10(1+)$ \\
\hline 2 & $G+\operatorname{cocci}$ & Staphylococcus saprophyticus. & $09(1+)$ & $12(1+)$ \\
\hline 3 & $\mathrm{G}+$ cocci in chains & Streptococcus agalactiae & $10(1+)$ & $08(1+)$ \\
\hline 4 & G- coccobacilli & E. coli & $06(1+)$ & $04(1+)$ \\
\hline 5 & $\mathrm{G}+$,long filamentous rods & Lactobacillus fermentum. & $12(1+)$ & $16(1+)$ \\
\hline
\end{tabular}

Vaginal swabs were obtained from women of control group and processed. The table shows the percentages and semi-quantitative grading in parenthesis of isolated and identified microorganism from 20-30 years and 31-40 years of age ( $n=100$ for each group).

variation in terms of vaginal Lactobacillus species due to geographical region are reported [15].

There was no significant difference in isolation percentage of Candida sp from test and control group of women aged 20-30 years which is consistent with the findings of Peddie, et al., [16] who reported no significant difference in contraceptives user and non-users when $C$. albicans was investigated. On the other hand, statistically significant difference in Candida sp compared to control was observed in women of age group 31-40 years which is not in agreement with the same study [16]. This elaborates the importance of age factor as a crucial variable and determinant for vaginal ecology. An inverse relationship was observed between E. coli and Lactobacillus fermentum. in women aged 20-30 years which is consistent with the findings of Gupta, et al. [6] however, no such association was established in our study for the group of women aged 31-40 year. The limitations of our study include unavailability of cultureindependent isolation of the vaginal microbiota. Although other species of Lactobacilli have been used as probiotic against UTI [17], the higher number of vaginal microbial flora in presence of Lactobacilli fermentum amongst women using $\mathrm{HC}$ indicate diminished role of this species and this may pose increased risk of the development of UTI in contraceptive users compared to non-users particularly in women above 30 years of age. Among the premenopausal women aged 37 to 54 years, with a history of oral contraception, a modest increase in the UTI has recently been published [1].

It has been reported that beside the suppression of ovulation, other peripheral manifestations in contraceptive users are thickening of cervical mucus, change in muscle tone and cervical endometrium $[11,18]$. Although these parameters were not investigated in the present study, the findings indicate that these factors may have been the cause of colonization of vagina with altered, higher percentages of vaginal microbiota found in our study and that these effects may become more enhanced in the age above 30 years.
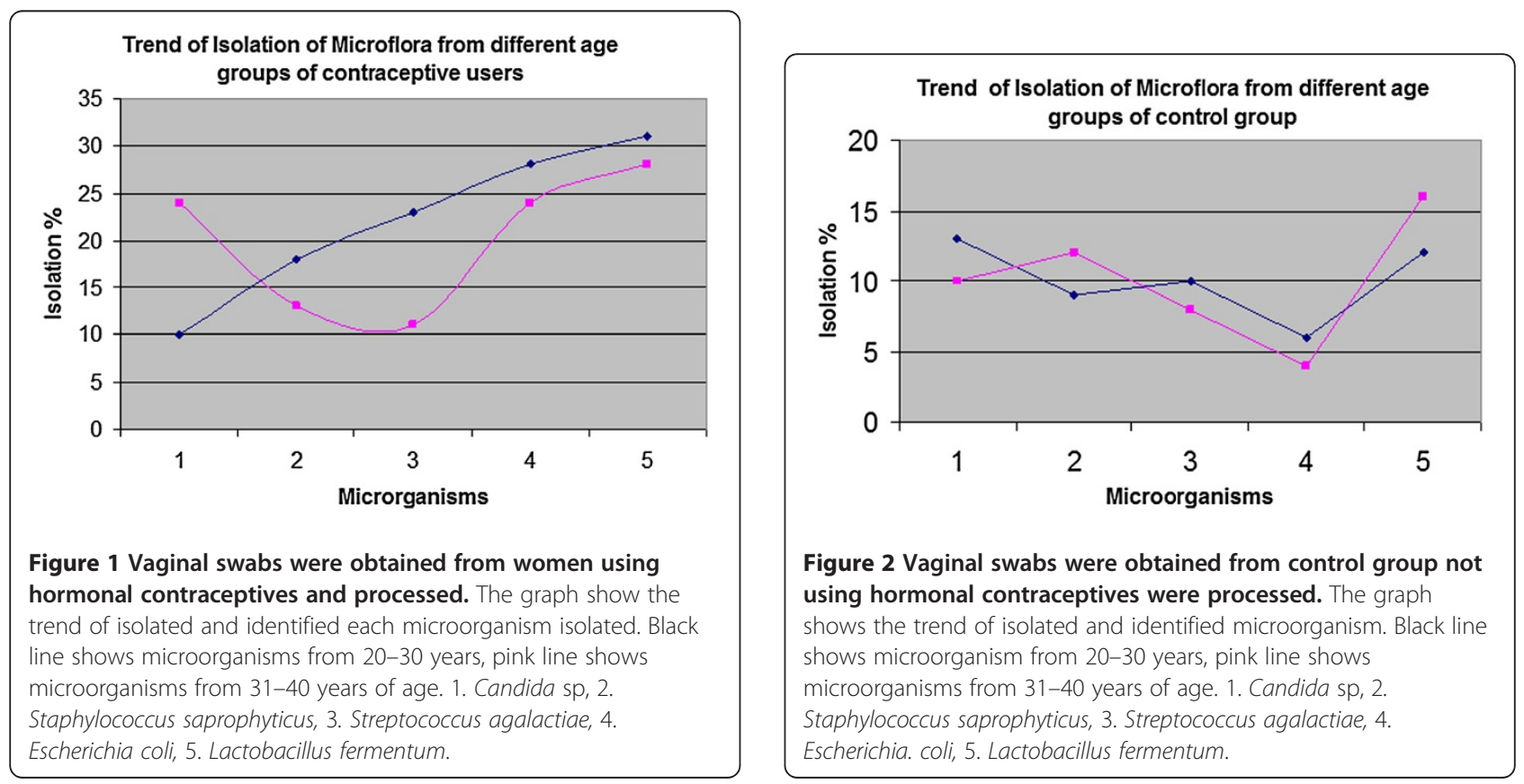


\section{Conclusion}

On the basis of the findings of this study it can be concluded that the age of a women using hormonal contraceptives is an important factor for the alteration in vaginal microbiota. The present study provides important information regarding the vaginal ecology of women using hormonal contraceptives particularly after 30 years of age. A greater attention to be paid to the family planning methods. The women using hormonal contraceptives may regularly be monitored for alterations in vaginal ecology in order to keep the UTI minimized.

\section{Competing interest}

The authors declare that they have no competing interests.

\section{Author's contributions}

YFK conceived and coordinated the study, SS processed the samples, NK collected the HVS samples. All authors read and approved the final manuscript.

\section{Acknowledgments}

We thank the Directorate of Research Shah Abdul Latif University Khairpur for funding this project and to Amjad A Mughal for technical assistance in Diagnostic and research center Department of Microbiology Shah Abdul Latif University Khairpur.

\section{Author details}

'Department of Microbiology, Shah Abdul Latif University, Khairpur, Sindh, Pakistan. ${ }^{2}$ Department of Pharmacology, Liaqat University of Medical and Health Sciences, Jamshoro, Sindh, Pakistan.

Received: 6 January 2012 Accepted: 9 August 2012

Published: 18 August 2012

\section{References}

1. Townsend MK, Curhan GC, Resnick NM, Grodstein F: Oral contraceptive use and incident urinary incontinence in premenopausal women. J Urol 2009, 181(5):2170-2175. doi:10.1016/j.juro.2009.01.040.

2. Steinauer JE, Waetjen LE, Vittinghoff E, Subak LL, Hulley SB, Grady D: Postmenopausal hormone therapy: does it cause incontinence? Obstet Gynecol 2005, 106:940.

3. Foxman B: Epidemiology of urinary tract infections: incidence, morbidity, and economic costs. Am J Med 2002, 113(1A):5. doi:10.1016/S0002-9343(02) 01054-9.

4. David AG, Kenneth FS: Nonspecific side effects of oral contraceptives: nocebo or noise? Contraception 2011, 83:5-9.

5. Population reference bureau 2011. www.pwdsindh.gov.pk.

6. Gupta K, Hillier SL, Hooton TM, Roberts PL, Stamm WE: Effects of contraceptive method on the vaginal microbial flora: a prospective evaluation. J Infect Dis 2000, 181:595-601.

7. Bauer AW, Kirby WM, Sherris JC, Turck M: Antibiotic susceptibility testing by a standardized single disc method. Am J Clin Pathol 1966, 36:493-496.

8. Balows A, Hauser WJ, Herrmann KL, Isenberg HD, Shadomy HJ: Manual of clinical microbiology. 5th edition. Washington, DC: American Society for Microbiology; 1991.

9. Cheesbrough M: District Laboratory Practice in Tropical Countries vol.2. New York: Cambridge University Press; 2006:62-70.

10. Vickers AA, Chopra I, O' Neil AJ: Intrinsic Novobiocin resistance in Staphylococcus. Antimicrob Agents Chemother 2007, 5:4484-4485.

11. Hooton TM, Scholes D, Hughes JP, Winter C, Roberts PL, Stapleton AE, Stergachis A, Stamm WE: A prospective study of risk factors for symptomatic urinary tract infection in young women. N Engl J Med 1996, 335:468-474.

12. Acton S, O'Meara YM: Urinary tract infection and contraceptive method. Irish Med J 1997, 90(5):176 [Pmid:9345824].

13. Dienye PO, Gbeneol PK: Contraception as a risk factor for urinary tract infection in Port Harcourt, Nigeria: a case control study. Afr J Prim Health Care Fam Med 2011, 3(1):1-4. doi:10.4102/phcfm.v3i1.207.
14. Reid G, Charbonneau D, Erb J, Kochanowski B, Beuerman D, Poehner R, et al: Oral use of Lactobacillus rhamnosus GR-1 and L. fermentum RC-14 significantly alters vaginal flora: randomized, placebo-controlled trial in 64 healthy women. FEMS Immunol Med Microbiol 2003, 35:131-134.

15. Pavlova SI, Kilic AO, Kilic SS, So JS, Nader-Macias ME, Simoes JA, et al: Genetic diversity of vaginal lactobacilli from women in different countries based on 16S rRNA gene sequences. J Appl Microbiol 2002, 92:451-459.

16. Peddie BA, Bishop V, Bailey RR, McGill HR: Relationship between contraceptive method and vaginal flora. Aust N Z J Obstet Gynaecol 1984 24(3):217-218.

17. Barbes C, Boris S: Potential role of lactobacilli as prophylactic agents against genital pathogens. AIDS Patient Care STDS 1999, 13:747-751.

18. Johnson JV, Grubb GS, Constantine GD: Endometrial histology following 1 year of continuous daily regimen of levonorgestrel $90 \mu \mathrm{g} / \mathrm{ethinyl}$ estradiol $20 \mu \mathrm{g}$. Contraception 2007, 75:23-26.

doi:10.1186/1471-2490-12-22

Cite this article as: Kazi et al:: Investigation of vaginal microbiota in sexually active women using hormonal contraceptives in Pakistan. BMC Urology 2012 12:22

\section{Submit your next manuscript to BioMed Central and take full advantage of:}

- Convenient online submission

- Thorough peer review

- No space constraints or color figure charges

- Immediate publication on acceptance

- Inclusion in PubMed, CAS, Scopus and Google Scholar

- Research which is freely available for redistribution 